\title{
Design of Straight-line Navigation System for Farm Machines Based on Mobile Internet Technology
}

\author{
Song Hualu ${ }^{1, a}$, Yan Yinfa ${ }^{1, b}$, Chen Chengcheng ${ }^{1}$, Zhang Xiaohui ${ }^{1}$, Li Fa-de ${ }^{1}$, \\ Sun Xuezhen ${ }^{2}$, Song Zhanhua ${ }^{* 1, c}$ \\ ${ }^{1}$ College of Mechanical and Electronic Engineering, Shandong Agricultural University, Tai'an \\ 271018, China; \\ ${ }^{2}$ College of Agronomy, Shandong Agricultural University, Tai'an 271018, China. \\ a songhualu@126.com, ${ }^{\mathrm{b}}$ sd28@163.com, ${ }^{\mathrm{c} *}$ (Corresponding author)songzh6688@163.com
}

Keywords: mobile internet technology, farm machines, straight-line navigation, inertial navigation technology, Android mobile terminal.

\begin{abstract}
In order to keep the farm machines straight in the agricultural production process, the straight-line navigation system for farm machines based on mobile internet technology and inertial navigation technology was developed. Straight-line navigation system consists of sensor subsystem and the Android mobile terminal. The sensor subsystem measures the deflection angle and uploads the angle to the Android mobile terminal via Wi-Fi. The Android mobile terminal that installed the straight-line navigation APP can accomplish real-time displaying the navigation data and setting the system parameters. The results indicate that the maximum offset error was less than $7.8 \mathrm{~cm}$ for straight trajectory tracking after the farm machine moved $50 \mathrm{~m}$ when the traveling speeds of the farm machine was not greater than $0.95 \mathrm{~m} / \mathrm{s}$. The straight-line navigation system can meet the requirements of agricultural production.
\end{abstract}

\section{Introduction}

Agriculture is the basic industry, and the meticulous agriculture is representing the direction of modern agriculture development, which is the focal point of modern agriculture research[1,2]. Farm machine automatic guidance is one of the important technologies for meticulous agriculture, which is the focal point of the research of the agricultural automatization and agricultural mechanization. It had a bright future in the fields of research and application.

There are many methods frequently used in farm machine's navigation, such as laser navigation, GPS navigation, electromagnetism navigation, ultrasonic navigation, vision navigation, etc[3,4]. But in actual experiment, there still some defects were founded in the navigation technique above, it can not meet the requirements of the practical application. On the other hand, the introduction of foreign products is very expensive, it is not convenient for extending them in some countries and regions.

Therefore, we design a straight-line navigation system for farm machines based on mobile internet technology and inertial navigation technology. This system is characterized with low coast, easy operating, and the practical applications shows that the system works satisfactorily. This system would decrease driver's fatigue and increase overall work productivity. All in all, the research of straight-line navigation system plays a highly important role in theory and practice.

\section{System Design}

Fig.1 illustrated the units of the straight-line navigation system, which is build-up by sensor subsystem and the Android mobile terminal. Sensor subsystem consists of 6 axis motion processing module, geomagnetic measuring module, power supply module, low-power Wi-Fi module and ARM microprocessor. 6 axis motion processing module realize the measurement of angular velocity and the geomagnetic measuring module realize the measurement of geomagnetic declination, both of them communicate the ARM microprocessor through IIC bus. According to the angular velocity 
and geomagnetic declination, the ARM microprocessor calculates the deflection angle, and uploads the angle to the Android mobile terminal via Wi-Fi which was set up by low-power Wi-Fi module. The power supply module is one important unit for the straight-line navigation system, which provides sufficient and stable source. And the Android mobile terminal that installed the straight-line navigation application can accomplish real-time displaying the navigation data and setting the system parameters.

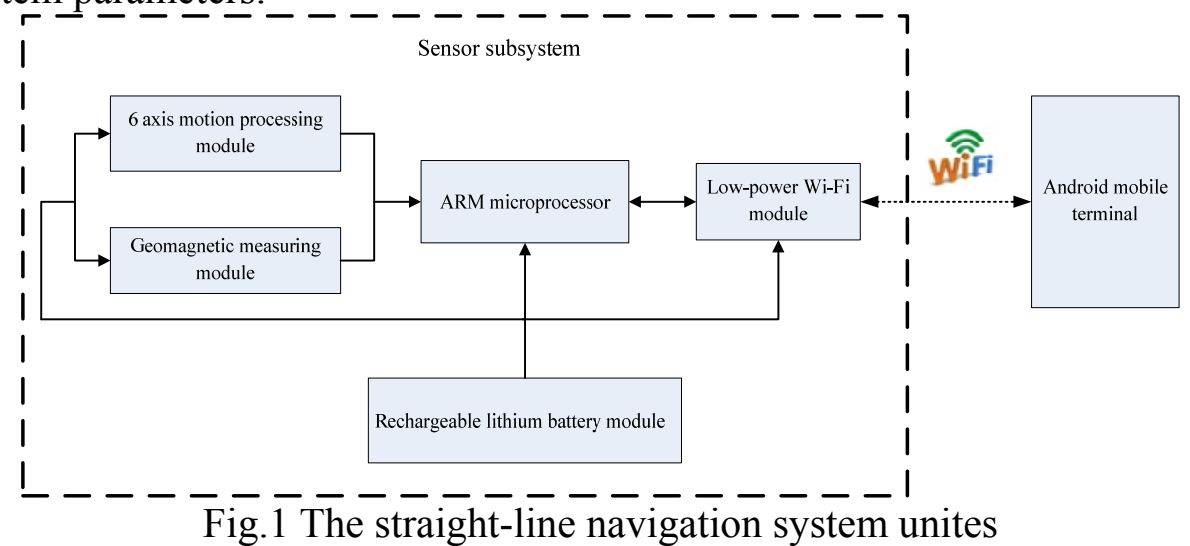

A. Hardware Design of Sensor Subsystem. The sensor subsystem is designed based on STM32F103C8T6, which provides minimizes overall system costs, the STM32F103C8T6 is perfect for general applications with low-power and high cost performance microcontroller solution in a small embedded application system. We also designs its peripherals hardware circuit to play its best performance, such as CAT811S monitoring circuitry, voltage regulator circuit, LED indicator and so on. The Fig. 2 shows the ARM microprocessor and peripheral circuits.
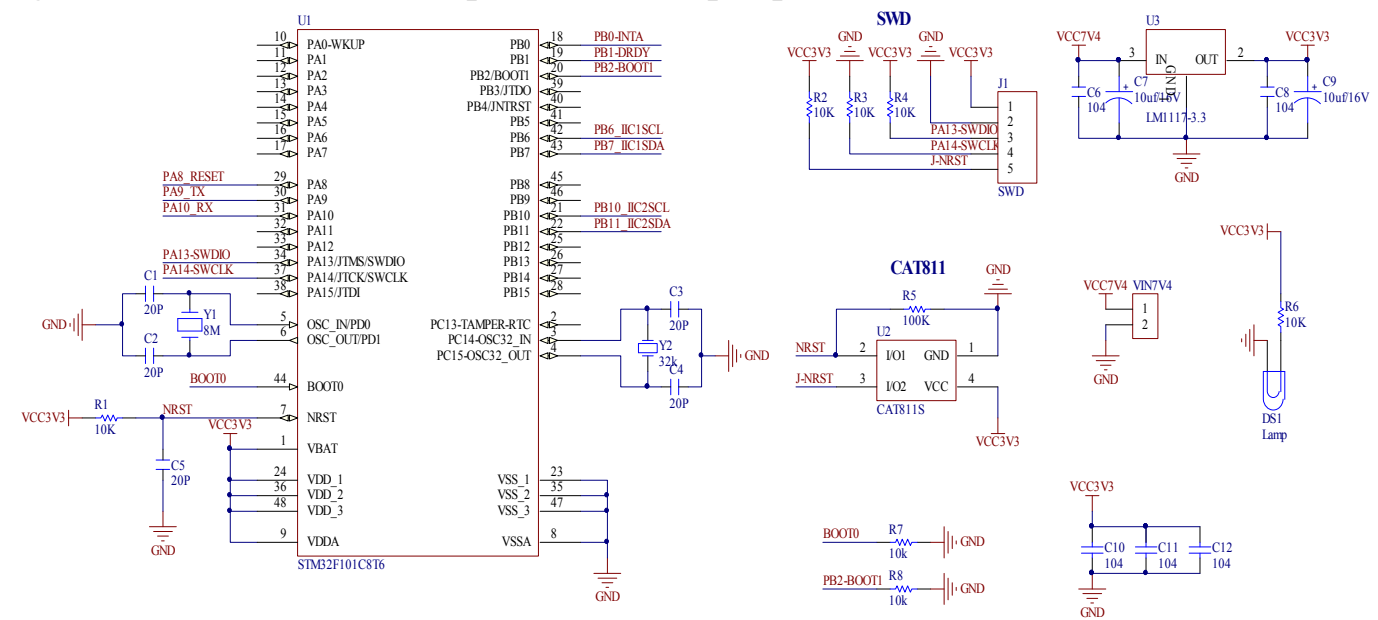

Fig. 2 The ARM microprocessor and peripheral circuits

B. 6 Axis Motion Processing Module and Geomagnetic Measuring Module Design. The MPU6050 is the core part of the 6 axis motion processing module, which is designed to measure angular velocity. The MPU6050 has a Digital Motion, a 3-axis MEMS accelerometer, and an embedded 3-axis MEMS gyroscope. It features three 16-bit ADCs for digitizing the accelerometer outputs and three 16-bit analog-to-digital converters (ADCs) for digitizing the gyroscope outputs. For precision tracking of both fast and slow motions, the parts feature a user-programmable accelerometer full-scale range of $\pm 2 \mathrm{~g}, \pm 4 \mathrm{~g}, \pm 8 \mathrm{~g}$, and $\pm 16 \mathrm{~g}$ and a user-programmable gyroscope full-scale range of $\pm 250, \pm 500, \pm 1000$, and $\pm 2000 \%$ sec.

The HMC5883L is the core part of the geomagnetic measuring module, which is designed to measure geomagnetic declination. The HMC5883L is a multi-chip, surface-mount module designed for magnetic sensing with a digital interface for applications such as low-cost magnetometry.

According to the angular velocity and geomagnetic declination, the ARM microprocessor calculates the deflection angle with the method of fusion algorithm[5,6]. As shown in Fig.3, the ARM microprocessor communicates with the MPU6050 and the HMC5883L through IIC bus. 


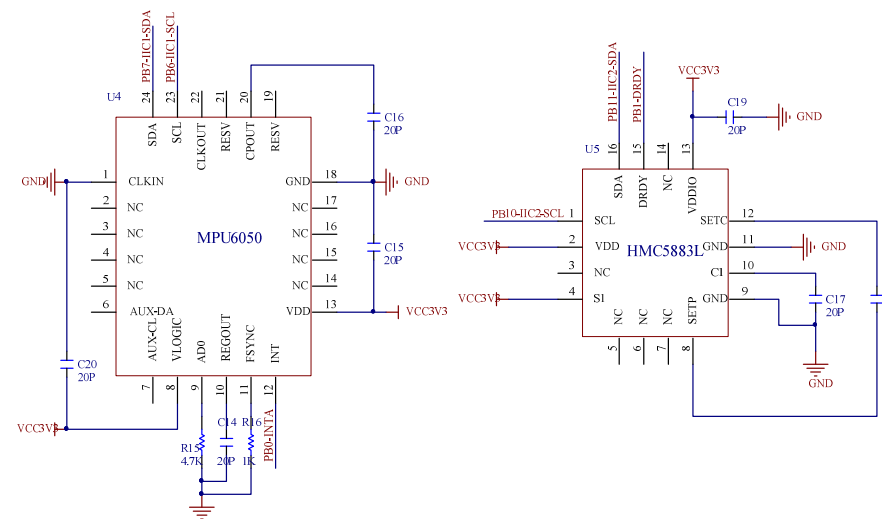

Fig. 36 axis motion processing module and geomagnetic measuring module

C. Low-power Wi-Fi Module. Low-power Wi-Fi Module allows the sensor subsystem to wirelessly connected to the Android mobile terminal. The Fig.4 shows that the module based on the ESP8266 Wi-Fi chip. The ESP8266 provides a network (IP) stack capable of both TCP and UDP. The ESP8266 is a complete and self-contained Wi-Fi network solution, which could run independently and could also be used as a slave carry on other host. In this paper, the ESP8266 work in the mode of server, set up a local area network (LAN) for Android mobile terminal connection. STM32F103C8T6 send data to ESP8266 through serial port, and then the ESP8266 would send the data via wireless network. In the same way, the data received via wireless network would be sent to STM32F103C8T6 by ESP8266 through serial port.

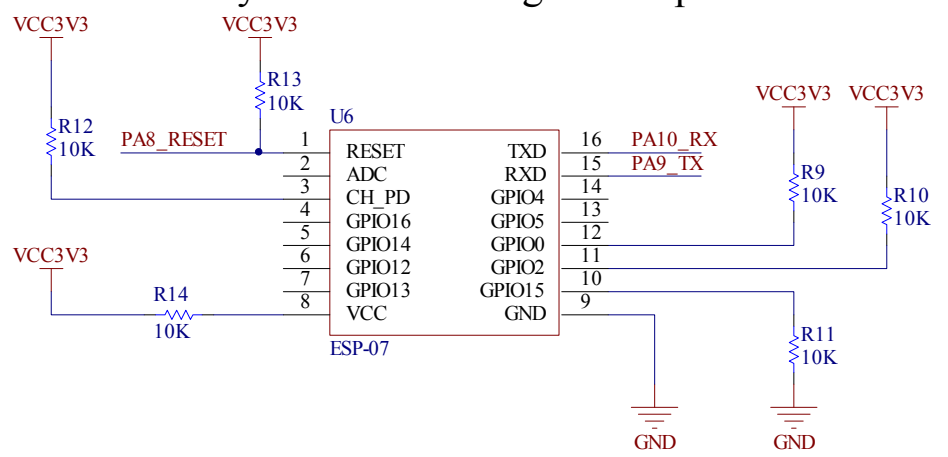

Fig.4 Low-power Wi-Fi module

D. Power Supply Module Design. Power supply module is consisted of one rechargeable lithium battery with output voltage $7.4 \mathrm{~V}$ and the management circuit which could provide overdischarge protection, overload protection and short circuit protection. The LM117 changes $7.4 \mathrm{~V}$ into $3.3 \mathrm{~V}$, for supply the sensor subsystem energy.

E. Software Design. The straight-line navigation system software mainly achieves deflection angle measurement and Wi-Fi communication. The STM32F103C8T6 drive the ESP8266 to open a TCP socket, set up a LAN server. In other words, the sensor subsystem is running in LAN server node mode. In the case of setting correctly the IP address and port address in the application which is installed in the Android mobile terminal, the Android mobile terminal could establish a connection to the LAN server, and then receive deflection angle from sensor subsystem.

The flow chart of sensor subsystem is depicted as shown in Fig.5. After the sensor subsystem is successfully initialized, the sensor subsystem will set up a LAN server. Then the sensor subsystem measures and calculates the deflection angle. The sensor subsystem will continuously upload data after the Android mobile terminal has successfully connected to the LAN server. 


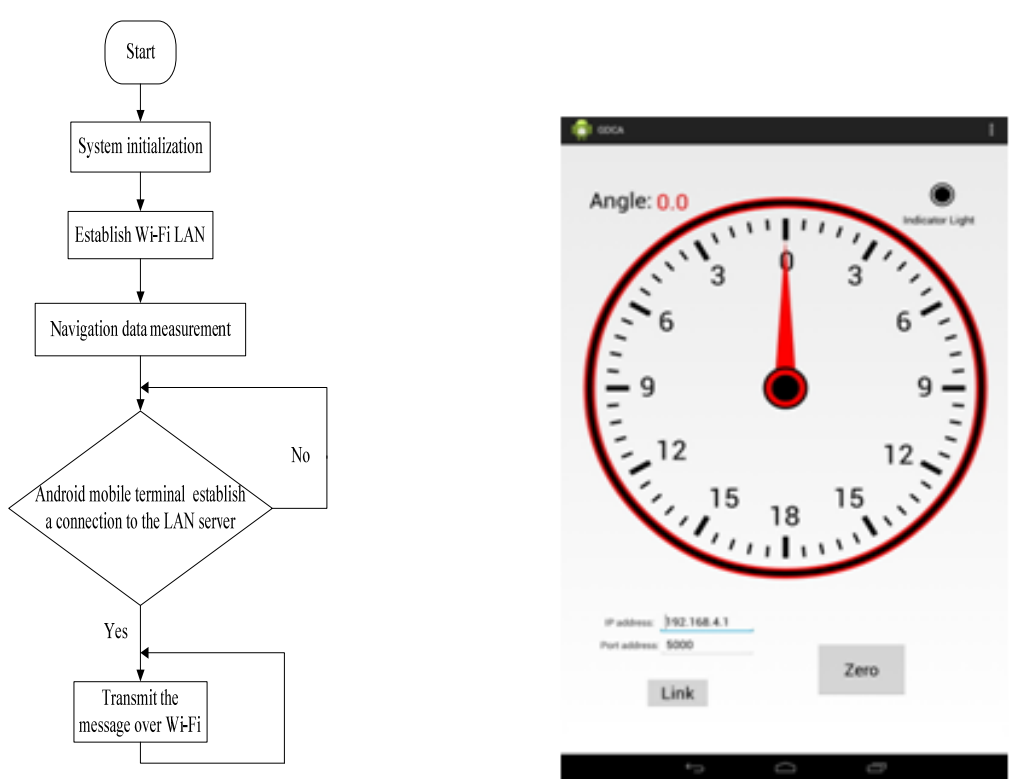

Fig. 5 The main UI of the straight-line navigation APP

The straight-line navigation APP is developed based on Android Studio[7,8]. Fig. 5 shows the main UI of the straight-line navigation APP. Input IP address, port address, and click "Link" button, then the Android mobile terminal will establish a connection to the sensor subsystem and the indicator light turns red. There is a dial on the application's display carried one pointer indicating the deflection angle. Meanwhile there is a window which could display the deflection angle in digital. No matter the farm machine now shows the course angle is how much, clicking "Zero" button, this course will be set to zero for farm machine. By watching the deviation from zero, driving direction will be adjusted real-time by the driver to keep the farm machine go straight.

\section{Experiment}

M254-E tractor made by Foton Lovol was used as test platform. The sensor subsystem was put into a seal box which was mounted on the front traction frame, and the Android mobile terminal was placed in the cab. The scriber, fastened to the bottom of the front traction frame, was used to record the running track. A tight nylon rope, with length up to $100 \mathrm{~m}$, marked the navigating datum line. The statistics and analysis of tractor on the situation of changes of straightness deviation were done in different speed, different driving range. The data of straightness deviation are shown as Table.1.

Table.1 Experimental data

\begin{tabular}{cccc}
\hline $\begin{array}{c}\text { Driving range } \\
\text { number }\end{array}$ & Speed $/ \mathrm{m} \cdot \mathrm{s}^{-1}$ & $\begin{array}{c}\text { Sample point } \\
\text { number }\end{array}$ & $\begin{array}{c}\text { Straightness deviation } \\
/ \mathrm{cm}\end{array}$ \\
\hline \multirow{3}{*}{ L1 } & & 1 & 4.5 \\
& 0.55 & 2 & 5.0 \\
& & 3 & 2.5 \\
& & 4 & 0 \\
L2 & & 5 & 3.0 \\
& 0.75 & 2 & 6.0 \\
& & 3 & 7.5 \\
& & 4 & 2.5 \\
& & 5 & 8.0 \\
L3 & 1 & 7.0 \\
\hline & & 2 & 7.5 \\
& 0.95 & 3 & 4.0 \\
& & 4 & 4.5 \\
& & 5 & 2.5 \\
& & & 6.5 \\
\hline
\end{tabular}




\section{Summary}

The field test and operation show that the system is reliable and can meet the design requirements. The maximum offset error was less than $7.8 \mathrm{~cm}$ for straight trajectory tracking after the farm machine moved $50 \mathrm{~m}$ when the traveling speeds of the farm machine was not greater than $0.95 \mathrm{~m} / \mathrm{s}$. In a word, the straight-line navigation system could meet the requirements of agricultural production quite well.

\section{Acknowledgements}

This work is supported by Science Foundation for Post-doctoral Scientists of Shandong Agricultural University "The Key Technology Study of Precision Direct Seed-metering Machinery for Cotton" and Intelligent Agricultural Equipment Research and Development Projects of Shandong Agricultural University in 2015 "The Design of Straight-line Navigation System for Farm Machines Based on Mobile Internet Technology". Thanks for the support provided by Professor Zhang Xiaohui (Cotton Innovation Team of Shandong Morden Agriculture Industry Technology System: SDAIT-07-011-10) and Professor Li Fa-de (Silkworm Innovation Team of Shandong Morden Agriculture Industry Technology System). Note: Song Zhanhua is the corresponding author of this paper.

\section{References}

[1]Diao Zhihua, Wang Huidan, Song Yinmao. Research of farm machinery navigation algorithm baded on machine vision[J]. Journal of Agricultural Mechanization Research, 2015, 37(2), pp. 35-39.

[2] Ma Hongxia, Ma Mingjian, Ma Na,et al. Baseline recognition based on Hough transform in the vision navigation ofagricultural vehicles[J]. Journal of Agricultural Mechanization Research, 2013, 35(4), pp. 37-43.

[3] Wang Xinzhong, Han Xu, Mao Hanping, et al. Navigation line detection of tomato ridges in greenhouse based on least square method[J]. Transactions of the Chinese Society for Agricultural Machinery, 2012, 43(6), pp. 161-166.

[4] Han Y H, Wang Y M, Kang F. Navigation line detection based on support vector machine for automatic agriculture vehicle[C]// International Conference on Automatic Control and Artificial Intelligence (ACAI 2012), Xiamen, 2012, pp. 1381-1385.

[5] Zhu Jianliang, Wang Xingquan, Wu Panlong, et al. Three-dimensional magnetic compass error compensation algorithm based on ellipsoid surface fitting[J]. Journal of Chinese Inertial Technology, 2012, 20(5), pp. 562-566.

[6] Long Dafeng, Liu Jun, Zhang Xiaoming, et al. Triaxial gyroscope fast calibration method based on ellipsoid fitting[J]. Chinese Journal of Scientific Instrument, 2013, 34 (6), pp. 1299-1305.

[7] Yang Linnan, Gao Lutao, Lin Ersheng, et al. Intelligent diagnose system of diseases and insect pests in sweet corn based on mobile terminal with Android system[J]. Transactions of the Chinese Society of Agricultural Engineering (Transactions of the CSAE), 2012, 28(18), pp. 163-168.

[8] Han Di, Pan Zhihong. Somatic Operation based on sensor of android mobile devices[J]. Journal of South China University of Technology: Natural Science Edition, 2012, 40(9), pp. 75-80. 